\title{
La planificación de estrategias de enseñanza en un entorno virtual de aprendizaje
}

Fecha de recepción : 2020-07-30 • Fecha de aceptación: 2020-12-15 • Fecha de publicación: 2021-10-01

\author{
Betty Pastora Alejo ${ }^{1}$ \\ Universidad Tecnológica Israel \\ bpastora@uisrael.edu.ec \\ https://orcid.org/0000-0002-9837-3264 \\ Arian Fuentes Aparicio² \\ Colegio Bilingüe Almirante Nelson \\ arian.fuentes@ueanan.edu.ec \\ https://orcid.org/0000-0002-2633-2244
}

\section{Resumen}

En el presente artículo se define como objetivo general analizar la importancia que tiene la planificación de estrategias de enseñanza en un Entorno Virtual de Aprendizaje (EVA) que en la actualidad se incrementa debido a la influencia de la era digital, donde la educación convencional presencial quedará invisibilizada por los avances de las Tecnologías de la Información y Comunicación (TIC), lo que supone nuevas formas de abordar el acto educativo para desarrollar en el alumnado el pensamiento computacional y de resolución de problemas, asumir una postura autocritica, y critica frente a la realidad social. Por otra parte, el proceso metodológico se abordó a partir del paradigma cuantitativo mediante la investigación descriptiva con un diseño documental. Además, se aplicó una encuesta a los profesores de la Universidad Tecnológica Israel del Distrito Metropolitano de Quito, Ecuador, en el Lapso Académico 2020/A, que permitió el análisis estadístico y de contenido. De esta manera, se concluye que la planificación de estrategias en un ambiente virtual exige en el profesorado la organización minuciosa para administrar a tiempo el aula virtual, seleccionar los recursos y medios tecnológicos que más se adapten a las necesidades o requerimientos del aprendiz, con la finalidad de propiciar la autogestión del aprendizaje independiente y el trabajo colaborativo entre estudiante/ 
estudiantes desde un enfoque constructivista en una experiencia contextualizada, interactiva y de responsabilidades compartidas.

Palabras clave: planificación, entorno virtual de aprendizaje, estrategias, era digital.

\begin{abstract}
This article defines as a general objective to analyze the importance of planning teaching strategies in a Virtual Learning Environment (VLE) that is currently increasing due to the influence of the digital age, where conventional classroom education will be invisible due to the advances of Information and Communication Technologies (ICT), which implies new ways of approaching the educational act to develop in students the computational thinking and problem solving, assume a self-critical position, and criticize the social reality. On the other hand, the methodological process was approached from the quantitative paradigm through descriptive research with a documentary design. In addition, a survey was applied to the professors of the Universidad Tecnológica Israel of the Metropolitan District of Quito, Ecuador, in the 2020/A academic year, which allowed for statistical and content analysis. In this way, it is concluded that planning strategies in a virtual environment requires teachers to organize themselves carefully in order to administer the virtual classroom on time, select the resources and technological means that best suit the needs or requirements of the learner, in order to promote self-management of independent learning and collaborative work between students/students from a constructivist approach in a contextualized, interactive experience with shared responsibilities.
\end{abstract}

Keywords: planning, virtual learning environment, strategies, digital age. 


\section{Introducción}

La era digital en contante trasformación y desarrollo requiere de una educación que permita a los estudiantes ampliar sus habilidades, y destrezas para responder a los requerimientos de un mercado laboral que está conectado con el avance tecnológico. Entonces, en el proceso educativo, las herramientas tecnológicas sirven como un medio pedagógico que contribuyen a potenciar el pensamiento crítico de resolución de problemas, aumentar las competencias computacionales, la participación activa de manera individual y el trabajo colaborativo grupal.

Es por ello que, los Entornos Virtuales de Aprendizaje (EVA) aportan mejoras a la calidad y variedad en la enseñanza-aprendizaje que no se consiguen utilizando los métodos convencionales (Britain \& Liber, citado por Scheuermann \& Barajas, 2003). Por lo tanto, estas plataformas educativas dan soporte a la educación superior actual (Fernández, 2007). Cabe destacar que, la gran mayoría de las instituciones escolares se vieron en la necesidad de incorporar el E-learning para impartir una enseñanza bajo la modalidad semipresencial y a distancia.

Asimismo, se puede indicar que los medios tecnológicos conectan a un mayor número de usuarios o estudiantes que en la instrucción presencial (Mortera, 2007). Para que esto sea posible, es necesario alejarse de los métodos didácticos clásicos a la hora de adaptar las actividades de enseñanza-aprendizaje, ya que tales métodos suelen basarse en una concepción conductista (González, 2002). Del mismo modo, emplear recursos multimedia, las clases magistrales grabadas, las teleconferencias que acrecientan la calidad educativa y la autonomía del aprendiz.

No obstante, en la planificación de estrategias de enseñanza se deben analizar las posibles dificultades que se puedan presentar en cualquier tarea; los conocimientos previos del alumno, las condiciones en que se llevará a cabo y el contexto específico para el que se ha diseñado (González, 2014). Situación que demanda de un alto sentido de responsabilidad por parte del profesorado para administrar a tiempo su aula con esmero y dedicación, que a la vez proyecta el toque particular de cada docente en la medida organizativa de las actividades y los recursos que implementará en ese espacio virtual.

Así pues, en la gestión académica, lo importante "consiste en que la modalidad de trabajo elegida sea la más idónea para el contenido concreto que se va a trabajar, el objetivo que se persigue, ya que a menudo lo que sirve a unos fines está contraindicado para otros" (González, 2014, p. 60). Conviene puntualizar que, en ese acto de planificación, prevalezca un alto desempeño profesional con rigor académico, condiciones que le solicitan al profesorado obtener conocimientos de estrategias interactivas que se ajusten a los contenidos instruccionales y a la comunicación sincrónica o asincrónica.

En efecto, la planificación de un Entorno Virtual de Aprendizaje (EVA) sugiere una estructura orientativa de los principios que guían la enseñanza de manera efectiva y para comprender que cada asignatura es diferente (Buontempo, 2017). Por eso, incluye objetivos y contenidos a enseñar a partir de un enfoque constructivo, integrador e interactivo donde el alumnado pueda acceder al conocimiento de un área específica y contar con un material de trabajo, así como de las instrucciones en todas las actividades a realizar. A su vez, "el grado de estructuración del 
contenido en un aprendizaje significativo además del establecimiento de conexiones entre la nueva información y el conocimiento previo es que éste se presente muy organizado y que dicha organización sea evidente" (Ausubel et al., 1983, p. 64).

Del mismo modo, en esa planificación es posible utilizar una variedad de interacciones de carácter interpersonal como las comunicaciones uno-a-uno en las consultas personales al profesor para la resolución de dudas, aclaraciones individuales y en las comunicaciones entre estudiantes; comunicaciones uno-a-muchos a través de lecturas, enunciados de ejercicios, recomendaciones generales; y comunicaciones muchos a-muchos en debates, discusión de casos, simulaciones, «brainstormings», entre otros (Mestre et al., 2007, p. 6).

Por consiguiente, el profesor como un mediador en la enseñanza-aprendizaje tiene que programar experiencias enriquecedoras en el aprender haciendo. Conforme a ello, se hace necesario pasar del docente transmisor de información a facilitador de conocimientos significativos; de alumno pasivo a estudiante activo; del aula fija al aula móvil y del libro de texto al multimedia (Real, 2011). Estas consideraciones son demostrativas al momento de planificar un entorno virtual donde no existen barreras de tiempo ni de espacio; comunicación sin fronteras, una forma diferente de atender a las necesidades individuales del estudiantado, compartir experiencias en un ciberespacio en el que todos aprenden, fomentar el aprendizaje cooperativo en el cual los discentes asuman el protagonismo en la autogestión de su propio aprendizaje.

Por tal motivo, la Universidad Tecnológica Israel, en Quito-Ecuador, en el Lapso Académico 2020/A, estableció una nueva Estructura tecno-pedagógica en el Entorno Virtual de Aprendizaje (EVA) para lograr en la comunidad estudiantil un Proceso de Aprendizaje Significativo (PAS) mediante la metodología Flipped Classroom, y la Metodología PACIE, donde la instrucción directa se resuelve desde el espacio de aprendizaje colectivo e individual en el que la figura del profesor esta ideada para guiar a los estudiantes en un espacio interactivo y dinámico (Instructivo de estructura tecno-pedagógica de aula virtual en el entorno virtual de Aprendizaje "EVA" UISRAEL 2020).

De allí que, la metodología PACIE, permite el uso de las TIC como un soporte a los procesos de aprendizaje y autoaprendizaje dando realce al esquema pedagógico de la educación real (Camacho, 2009). Respecto a esto, se puede decir que la metodología PACIE, en el Entorno Virtual de Aprendizaje (EVA) accede a planificar diversas estrategias de enseñanza basada en las directrices metodológicas implícitas en el Modelo Educativo UISRAEL 2016-2020. Por nombrar algunos ejemplos:

De rebote: conlleva a la participación individual en una postura autocritica del estudiante en el que estos demuestran la comprensión de la información que se adjunta en los documentos de referencias a través de un archivo o enlace. Se recomienda crear cuestionarios cortos tipo Kahoot o chat.

De construcción: genera un aprendizaje colaborativo para que los participantes respondan preguntas relacionadas con el tema a tratar en clases. Se exhorta emplear foros de discusión, lecciones o debates. 
De comprobación: implica tareas a modo de indicador de que están comprendiendo el material trabajado. Para hacerlo se proponen cuadros comparativos, organizadores gráficos, ensayos, resumen, estudios de casos integrador, resolución de ejercicios.

En consecuencia, al planificar estrategias en un Entorno Virtual de Aprendizaje (EVA), se deben considerar las variadas opciones en cuanto a actividades y recursos didácticos que se consiguen utilizar dentro de una estructura tecno-pedagógica con la intención de que los estudiantes desarrollen competencias cognitivas, actitudinales y el pensamiento crítico-reflexivo frente a un tema en cuestión (Instructivo de estructura tecno-pedagógica de aula virtual en el entorno virtual de Aprendizaje "EVA" UISRAEL 2020).

En este sentido, las TIC ofrecen a los docentes la posibilidad de replantear las actividades tradicionales de enseñanza para ampliarlas con nuevas actividades, recursos y estrategias de aprendizaje desde un enfoque constructivista, apoyado en el uso de herramientas informáticas (Collins, 1997). Ahora bien, la implementación de estas herramientas educativas demanda en el profesorado poseer competencias digitales básicas para la administración de los entornos virtuales.

Por esta razón, el profesor universitario debe mantenerse actualizado a la par de las innovaciones de las tecnologías. Aunado a que, en la sociedad contemporánea los estudiantes son considerados nativos digitales. Conviene acotar la importancia de las competencias digitales (CD), siendo "un conjunto de valores, creencias, compromisos, conocimientos, capacidades y actitudes que habría de adquirir, y en las que crecer para aportar su cuota de responsabilidad a fin de garantizar una buena educación para todos" (Escudero, 2006, p. 46).

De ahí que, la presente investigación busca analizar la importancia de la planificación de estrategias de enseñanza en un entorno virtual para el desarrollo de las clases acorde a las exigencias de la educación actual en un aprendizaje activo, novedoso y en constante feedback con el apoyo de las herramientas telemáticas a partir de un enfoque constructivo e integrador que contribuya a orientar el proceso educativo en todas las fases implicadas que suponen modificar las estrategias de enseñanza.

\section{Los entornos virtuales de enseñanza-aprendizaje}

El avance acelerado de la Tecnología de la Información y Comunicación (TIC) han generado múltiples beneficios a la colectividad, en especial en el ámbito educativo vinculado con los intereses y necesidades del educando a la vez dejando atrás el viejo modelo educativo donde el docente era un mero transmisor y expositor de información. Circunstancias que exigen al profesorado sólidos conocimientos en áreas específicas, habilidades investigativas, manejo de grupos, comunicación eficiente, administración factible del tiempo, capacidad autocritica y competencias computacionales.

Por eso, los ambientes de aprendizaje no se circunscriben al espacio escolar más bien se trata de aquellos espacios en donde se crean las condiciones para que el participante se apropie de 
nuevos conocimientos, experiencias y elementos que le generen procesos de análisis, reflexión y apropiación (Mentre et al., p. 14). Cabe puntualizar que, una de las bondades de las TIC está relacionada con la conectividad que facilita acceder a la información de forma rápida y hacer uso de cualquier recurso multimedia. Por lo demás, economiza esfuerzo y tiempo a través del uso de internet (Fernández et al., 2019).

Desde el surgimiento de los entornos virtuales de enseñanza se ha buscado la manera de vincular en el mismo todos los aspectos informáticos incorporado la multimedia como imagen, sonido, videos, otros. De la misma manera, tomar en consideración los cinco componentes principales que le conforman: el espacio, los estudiantes, los tutores, los contenidos educativos y los medios (Mentre et al., p. 16). Por ello, la planeación de la enseñanza en línea presenta ciertas falencias por parte del profesorado puesto que algunos continúan con la obsesión por la transmisión de contenidos, tendencia al uso de metodologías de naturaleza conductista, preferencia a la evaluación sumativa olvidándose en muchos casos del análisis de los procesos de construcción del conocimiento; excesivo descuido en el diseño de estrategias instructivas (Carrillo, 1995).

De este modo, la planificación de un entorno virtual de enseñanza conlleva a implementar todos los componentes del sistema didáctico (Mentre et al.). Situación que requiere la estructuración correcta del contenido a mostrar y que el alumno pueda apropiarse de los contenidos plasmados en el mismo. Concierta destacar la necesidad de realizar una alfabetización digital en la adquisición de nuevas habilidades para el entendimiento y la interacción con las tecnologías, es decir, se precisa el desarrollo de competencias para utilizarlas de forma eficaz, responsable e inteligente (Fernández et al., 2019).

En corolario, el profesorado deberá tener en cuenta concretar las ideas que se desea impartir; planificar situaciones para que los estudiantes socialicen entre ellos, aclaren inquietudes, argumenten sus puntos de vistas; vivencien experiencias asociadas con la cotidianidad y aprenden uno de los otros. Sumado a trabajar en la medida en que el grupo se organiza y aprende alrededor de la consecución de un objetivo (Galvis, 1992). También, desarrollen interdependencia positiva, interacción, contribución individual, habilidades personales y de grupo (Guiza, 2011)

Entonces, la planificación de estrategias en un entorno virtual puede derivar de un nivel alto de satisfacción por parte de los participantes siempre que se tome en cuenta todos los componentes que conforman el proceso educativo con la incorporación de las TIC, que estará direccionado y centrado en el alumnado en un acompañamiento continuo, y secuencial para confrontar con éxitos los desafíos del aprender haciendo, y que no afecte la prosecución efectiva de sus estudios.

\subsection{Planificación de estrategias de enseñanza en un entorno virtual}

Para planificar un aula virtual se debe tener en cuenta el enfoque constructivista donde el profesor, se convierte en un facilitador en todo el proceso de enseñanza-aprendizaje. Este acto educativo implica saber que enseñar, con cuáles estrategias y recursos didácticos se abordará cada sesión de aprendizaje. Concomitantemente, conocer las características particulares de cómo aprende el 
estudiante en sus estilos de aprendizaje y especial cuidado en preparar el aula desde un espacio de interacción agradable.

De este modo, el docente con una postura constructivista conduce las clases para permitir que los estudiantes construyan el conocimiento a partir de técnicas o métodos que generen la participación activa en un pensamiento crítico-reflexivo (Serrano \& Pons, 2011). Para ello puede crear su aula de clases en cada tema por encuentro en un lapso académico, que variará de acuerdo con el sistema de gestión de aprendizaje que utilice, por ejemplo, Blackboard, Moodle, Google Classroom o Canvas (Plataforma de ciencias del aprendizaje).

Por eso, al planificar cuidadosamente el aula virtual permitirá que los estudiantes fomenten el pensamiento transdisciplinario, prepararse antes de asistir a las clases, revisar de manera repetida las clases grabadas, cumplir a tiempo con todas las actividades o tareas programadas según calendario académico, revisar las presentaciones de las diapositivas pre clase, la asistencia a clases y entregar las tareas de comprobación en el momento solicitado.

Con relación a la ejecución de tareas grupales, es importante promover el logro de objetivos cualitativos más ricos en contenido, pues reúne propuestas y soluciones de varias personas del grupo, aumenta el aprendizaje de cada quien debido a que se enriquece la experiencia de aprender, incrementa la motivación por el trabajo individual y grupal, puesto que hay una mayor cercanía entre los miembros y compromiso con todos; aumenta la cercanía y la apertura. En el plano personal mejora las relaciones intergrupales; acrecienta la satisfacción por el propio trabajo y se valora el conocimiento como un aporte de los demás miembros del grupo; disminuye los sentimientos de aislamiento y el temor a la crítica. Amplia la retroalimentación permanente (Mestre et al, 2007).

Por otro lado, para la implementación de un Entorno Virtual de Aprendizaje (EVA) se debe considerar la metodología PACIE, que admite el uso de las TIC, como un soporte a los procesos de aprendizaje y autoaprendizaje, en el que se esquematiza los aspectos tecno-pedagógico de la educación virtual y/o distancia. El nombre PACIE, es el resultado de las iniciales de cada uno de los procesos secuenciales en los que se fundamenta esta metodología: $(P)$ Presencia, $(A)$ Alcance, $(C)$ Capacitación, (I) Interacción y $(E)$ E- learning (Camacho, 2009) que permite manejar de una mejor manera el proceso de transición, tanto en los espacios presenciales cotidianos como en los institucionales, de guiar por internet el proceso de enseñanza-aprendizaje con la utilización de recursos didácticos usados en clase (Camacho, 2009).

En efecto, toma en cuenta la motivación y el acompañamiento, la calidad y calidez humana versus la cantidad y frialdad; incorpora la comunicación y presentación de la información; los procesos sociales que apuntalan la criticidad y el análisis de los datos que acceda construir conocimientos mediante la interacción, y el intercambio de experiencias educativas para informar, exponer, crear, colaborar en un espacio interactivo donde el profesorado se convierte en un mediador para compartir información y conocimientos (Camacho, 2009).

Por lo tanto, la metodología PACIE, requiere de un diseño institucional con una imagen corporativa de estilo y formato para que los estudiantes al momento de ingresar al campus virtual encuentren 
recursos atractivos de la web 2.0, como animaciones, videos, que inviten a desarrollar la imaginación, la creatividad y la estimulación de los participantes en la autogestión del aprendizaje, con instrucciones precisas que soporten el trabajo individual y grupal donde se evidencie la calidad en las actividades, y discusiones propuestas (Camacho, 2009).

En este sentido, la administración de entornos virtuales requiere de una planificación organizada y secuencial de los contenidos a tratar en los diferentes encuentros de clases, delimitando cada objetivo de instrucción, los recursos didácticos, los criterios de evaluación con sus respectivas rubricas todo ello para lograr las actividades de inicio, desarrollo y cierre. Asimismo, tener presente los momentos de la comunicación sincrónica y asincrónica que supone un cambio significativo en la forma de enseñar-aprender bajo la modalidad en línea.

Como es evidente, el rol del profesor estará mediado en un buen o no aceptación de su desempeño profesional. Por lo tanto, se requiere la capacitación constante del profesorado para minimizar las dificultades que se le puedan presentar por la falta de competencias digitales, inexperiencia en la conducción grupal, déficit en la comunicación efectiva y el diseño de materiales acordes a cada ocasión. Mientras tanto, los estudiantes necesitan de un proceso de inducción para aprender administrar su aula virtual, poner en práctica el aprender haciendo en una proyección secuencial de sus nuevas capacidades, habilidades, destrezas y valores aprendidos en el proceso de enseñanza-aprendizaje.

El aprender haciendo implica aprender de verdad, apropiarse del conocimiento, construyéndolo paso a paso (Camacho, 2009). Por tal motivo, se demanda de estrategias de enseñanza en la cual se reciba la información en diferentes formatos ya sea con recursos multimedia o hipertextuales que produzcan el aprendizaje individual y colaborativo. A la vez, promueva el pensamiento computacional, el pensamiento crítico-reflexivo, la participación activa y el aprendizaje basado en problemas.

\subsection{Herramientas para la enseñanza en un entorno virtual}

El acto de enseñar en un Entorno virtual de aprendizaje (EVA) demanda de un proceso activo de las metodologías que se llevarán a la práctica. Por ende, las instrucciones deben orientar paso a paso para que los educandos gestionen el aprendizaje independiente. Sin obviar las tutorías académicas a fin de ofrecer el asesoramiento continuo para minimizar las limitaciones que suelen presentarse bajo esta modalidad.

Asimismo, coadyuvar a los estudiantes a transferir lo aprendido a situaciones reales y complejas. Esto aplica incluso para estudiantes sobresalientes. Es evidencia de un exacerbado malestar que se ha propagado por todo el nivel de educación superior, la manera cómo enseñamos y cómo aprenden los estudiantes favorece principalmente la memorización (Escamilla, 2019, p. 12). No obstante, el profesorado cuenta con una matriz de estrategias y recursos para realizar su práctica docente en aras de responder a los requerimientos actuales que consienta desarrollar en los estudiantes competencias disciplinares y transversales. 
Envuelve un aprendizaje basado en retos, flexibilidad en el cómo, cuándo y dónde se aprende, una vivencia universitaria memorable, profesores inspirados en aprender a reaprender, con trayectorias curriculares y contenidos más personalizados hacia el aprendizaje vivencial que reduce la brecha entre teoría-práctica que permite desarrollar competencias blandas ahora llamadas competencias poderosas (Escamilla, 2019, p. 13).

Asimismo, un aspecto importante en la autogestión del aprendizaje lo constituyen los recursos que sin estos sería casi imposible llevar a cabo con éxito la enseñanza virtual (Contreras et al., 1999). Por ende, facilita la creatividad, la flexibilidad de pensamiento para actuar en resolución de problemas, amplía la imaginación y la curiosidad. Entonces, uno de los mayores retos que están teniendo las universidades a nivel mundial es preparar a los alumnos para un trabajo cada vez más incierto. A la vista de la automatización y los progresos de la inteligencia artificial (Pelayo, 2019).

Por otra parte, en la planificación de estrategias en un entorno virtual se pueden utilizar diversos recursos didácticos que permiten el feedback inmediato con el soporte de las herramientas telemáticas y en el apoyo de la evaluación continua. También, mantener la información actualizada, diseñar materiales educativos, incrementar la productividad de participación del alumnado. En fin, la producción actualizada de los conocimientos en un aprender a conocer y aprender haciendo (Pelayo, 2019). A continuación, se describen algunas herramientas que pueden servir de soporte en el arte de enseñar:

Hipermedia: combina el hipertexto con los multimedia de manera que la información digital suele ser presentada en diferentes formatos como: texto, ilustraciones, sonidos, animaciones, videos (Mestre et al., 2007).

Navegación en red: exploración por la red siguiendo las relaciones que conectan los distintos nodos en los que se estructura el hipertexto para buscar, seleccionar y recuperar información (Mestre et al., 2007).

Interacción interpersonal: se puede emplear el correo electrónico y los debates en discusiones basadas en grupos (Mestre et al., 2007).

Las estrategias de tipo individualizado: entran en esta categoría los viajes de estudio, las visitas a lugares específicos, los juegos creativos, las conversaciones guiadas, informes, ensayos a modo de tareas (Escudero, 2008).

Los recursos institucionales: abarca las bibliotecas, los laboratorios, las aulas especializadas, las conferencias y los museos.

Los Foros de discusión: son panales en línea en los que los discentes pueden publicar un mensaje sobre un tema o pregunta y obtener respuestas de parte de otros participantes. Es un indicador de que los estudiantes están trabajando los materiales adjuntado en el aula virtual. Propicia el aprendizaje colaborativo (Plataforma de ciencias del aprendizaje). 
Mini bibliotecas: colecciones en línea que consisten en una variedad de enlaces a artículos de revistas, videos, podcasts, sitios web, libros electrónicos, ensayos, monografías. Ayuda a aumentar la motivación del aprendiz, enriquecer la lectura comprensiva y la discusión en clases (Plataforma de ciencias del aprendizaje).

Los portafolios electrónicos: adaptaciones digitales de los trabajos realizados por clases. Aprueba visibilizar la progresión de su propio crecimiento, las dificultades presentadas y mejorar su proceso formativo. Además de que fomenta el compromiso y la motivación al logro.

Pruebas frecuentes automatizadas: son evaluaciones que tienen poco impacto en la calificación final de los estudiantes. Contribuye a mejorar la retención de la información mediante la práctica de recuperación, reducir las brechas de rendimientos de aquellos que están menos preparados en un tema, minimizar la ansiedad ante los exámenes parciales y finales (Plataforma de ciencias del aprendizaje).

Producción de un video corto: facilita la creación de un texto narrativo, relatos, cuentos, parábolas, analogías.

Live class: genera una enseñanza personalizada. Se pueden usar los videos tutoriales y cualquier recurso multimedia que proporcione una experiencia enriquecedora a los estudiantes (MCA School of Business \& Postgraduate, 2020).

Conferencias magistrales: tienen un alto impacto de rigor académico y científico con demostraciones prácticas que suelen ser grabadas para que el participante las emplee bajo su disponibilidad de tiempo y según sus requerimientos personales (UISRAEL, 2020).

El chat: propicia la administración del tiempo por parte del estudiante para participar en estos espacios de comunicación sincrónica con la finalidad de construir el conocimiento sobre una temática planteada.

Tutorías académicas: Consiste en una planificación previamente organizada para brindar asesorías individuales o grupales de apoyo en el proceso de formación académica.

Se deduce que el profesorado según su experiencia profesional y área de disciplina será el encargado de planificar las estrategias de enseñanza que más se adecuen a sus estudiantes para llevar a cabo una práctica docente con rigor académico y de excelencia educativa. Por tanto, se debe tener en cuenta la importancia que representa el marco relacional en el que se desarrolla la actividad docente y de aprendizaje. No se puede usar las mismas dinámicas o metodologías docentes en un espacio sincrónico o asincrónico (Mestre et al., 2007).

En consecuencia, la gestión docente en los entornos virtuales siempre se desarrollará a modo de complemento con el uso de las TIC, para facilitar aprendizajes independiente y colaborativo. Entonces, el profesor se convierte en un mentor de acompañamiento en actitud positiva y de alto impacto de creatividad en aras de orientar todo el proceso de formación integral de los estudiantes. 
Además, las instituciones educativas tienen la responsabilidad de acondicionar la infraestructura que permita la fácil vinculación con la tecnología de punta.

Por consiguiente, se debe realizar un plan de capacitación en la formación permanente del docente sin obviar que, en la era digital la información caduca rápidamente aunado a desarrollar las competencias para la comunicación efectiva, la toma de decisiones, la resolución de problemas técnicos, el manejo de grupo, el uso eficiente del tiempo. Sin embargo, "cada profesor tiene una forma distinta de llevar la clase, aplicar los métodos, elaborar el programa y de relacionarse con los estudiantes" (Bennett, 1979. p.2).

\section{Metodología}

La presente investigación se aborda desde el enfoque cuantitativo, que "es un proceso metódico y sistemático dirigido a la solución de problemas o preguntas científicas mediante la producción de nuevos conocimientos" (Hernández, et al. 2014, p. 37). Asimismo, se utilizó la investigación descriptiva que "consiste en la caracterización de un hecho o fenómeno, individuo o grupo con el fin de establecer su estructura o comportamiento" (Arias, 2006, p. 24).

Por ello, se utilizaron diferentes métodos de investigación que posibilitaron desarrollar un conjunto de procedimientos lógicos en aras de llevar a cabo las etapas de la investigación científica. También se consideró conveniente fundamentar el estudio a través del diseño documental, momento en el cual los investigadores asumieron que es un proceso basado en la búsqueda, recuperación, análisis, crítica e interpretación de la información que se obtiene en fuentes impresas o electrónicas para aportar nuevas ideas (Arias, 2006).

De acuerdo a esto, conllevó a consultar diversos autores que permitieron examinar las posturas teóricas en un aporte en el plano de la teoría sobre la temática abordada con la finalidad de demostrar la validez del análisis de contenido. Es por eso que, el estudio se realizó en la Universidad Tecnológica Israel del Distrito Metropolitano de Quito, en el Lapso académico 2020-A, con la aplicación de una encuesta a 7 docentes seleccionados de manera intencional.

En consecuencia, la encuesta "consiste en una técnica que pretende obtener información que suministra un grupo o muestra de sujetos acerca de si mismo, o en relación con un tema particular" (Arias, 2006, p. 72). Esta situación posibilitó recoger los datos para conocer el criterio de los profesores en cuanto a las estrategias de enseñanza que emplean en la planificación de su quehacer universitario. Además, las evidencias documentales, el análisis de contenido y estadístico permitieron dar cumplimiento a la matriz de objetivos de la investigación.

Siendo el general analizar la importancia que tiene la planificación de estrategias de enseñanza en un Entorno Virtual de Aprendizaje (EVA), acorde con los requerimientos de los estudiantes de la educación contemporánea.

Mientras que los especificios fueron: 
- Indagar las teorías que fundamentan la enseñanza en un Entorno Virtual de Aprendizaje (EVA).

- Describir las estrategias de enseñanza más utilizadas en el entorno virtual.

- Caracterizar la importancia de la planificación de estrategias de enseñanza en un Entorno Virtual de Aprendizaje (EVA).

\section{Resultados}

\section{Análisis de los resultados y discusión}

Las transformaciones de las TIC propiciaron en la educación contemporánea cambios significativos que conllevaron a modificar la instrucción tradicional con el propósito de responder a los requerimientos de los estudiantes considerados nativos de la cultura digital, lo que supuso en la educación superior implementar otras formas de conducir el proceso educativo, para la incorporación de entornos virtuales bajo la modalidad semipresencial y a distancia.

Estos cambios modificaron radicalmente las estrategias de enseñanza privilegiando una participación más activa del alumnado y centrada en el aprender a aprender. Condición que solicita al profesorado además de dominar el contenido curricular, tener conocimiento de los procesos implicados en el aprendizaje y requieren métodos flexibles adaptados a las necesidades individuales, y basados en el diálogo a fin de poder ofrecer la realimentación necesaria durante el proceso educativo donde las TIC juegan un rol importante para apoyar la gestión académica y las dificultades que suelen presentar los discentes en la adaptación de un entorno virtual (Escontrela \& Stojanovic, 2004).

Por ello, es importante preguntarse cómo educar en la era digital y con cuáles estrategias se puede fomentar el aprendizaje independiente y colaborativo, sustentado a partir del enfoque constructivista, que se define como el "desarrollo armónico e integral de las capacidades intelectuales, psicomotoras, aptitudinales, actitudinales del ser humano" (Pulgar, 2005, p. 19, citado por Ortiz 2005).

En este sentido, la administración de un entorno virtual permite al profesorado la utilización de diversas estrategias de enseñanza con el apoyo de las TIC, entre las que se pueden mencionar: los recursos audiovisuales, materiales digitalizados en PDF, animaciones, imágenes, sonidos, etc, que resultan atractivas, novedosas e interesantes, en el que los discentes se motivan, involucran en su propio aprendizaje y en el trabajo grupal. A la vez desarrolla habilidades, destrezas y el pensamiento computacional (González, 2014).

No obstante, las estrategias de enseñanza son procedimientos o recursos organizadores del conocimiento utilizados por el docente para promover aprendizajes significativos que desarrollen los procesos cognitivos, conductuales, sociales y afectivos de quien aprende. Partiendo de la idea fundamental de que el profesor es el mediador del aprendizaje; además de enseñar los contenidos de su disciplina, asume la necesidad de enseñar a aprender (Díaz \& Hernández, 1999). 
De este modo, se presentan los resultados del análisis estadístico -porcentual mediante la representación en tablas y figuras, con la finalidad de mostrar la práctica docente en la UISRAEL bajo el Entorno Virtual de Aprendizaje (EVA), que permite la reflexión en el uso de las tecnologías y las fortalezas de las estrategias de enseñanza en la gestión pedagógica actual que influye directamente en el aprendizaje de los estudiantes.

En la Tabla 1 se evidencia el grado académico de los profesores que conforman la Universidad Tecnologica Israel.

Tabla 1.

Grado académico de docentes

\begin{tabular}{|l|l|l|l|l|}
\hline Resultado & Grado & Maestría & Doctorado & Total \\
\hline $\mathrm{Fi}$ & 7 & 5 & 2 & 7 \\
\hline $\mathrm{F} \%$ & $100 \%$ & $71,43 \%$ & $28,57 \%$ & $100 \%$ \\
\hline
\end{tabular}

Fuente: elaboración propia

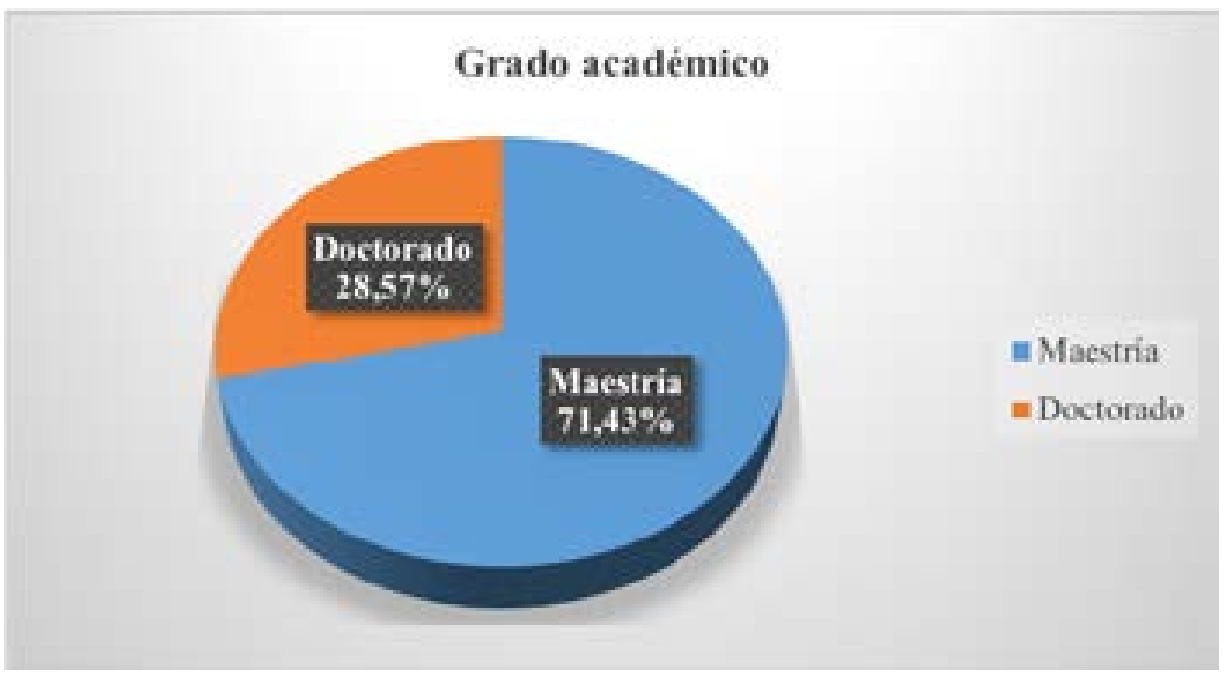

Figura 1. Grado Académico de Docentes

Fuente: elaboración propia

Como se puede evidenciar en la Figura 1, un $71,43 \%$ de los profesores cursaron estudios de postgrado en maestría mientras que, el $28,57 \%$, han logrado el grado académico de PhD. Los resultados demuestran que estos profesionales universitarios se mantienen actualizados.

Cabe destacar la importancia de los estudios de cuarto nivel, que constituye sin lugar a duda, el desarrollo profesional de los profesores para el perfeccionamiento de su gestión académica y de investigación que coadyuva a una educación superior de calidad.

En efecto, en un mundo globalizado las universidades, como espacio de formación profesional, producción y diseminación de conocimientos, tienen el gran reto de desarrollar el talento humano 
de excelencia y comprometido con la misión social de la educación superior en aras de elevar continuamente el nivel ideológico, político y técnico de los profesionales (Rodríguez, 2011 citado por Romero, 2017).

En la Tabla 2 se observa el uso de estrategias de enseñanza que fueron utilizadas en la plataforma EVA por los docentes.

Tabla 2.

Estrategias de enseñanzas en el Entorno Virtual de Aprendizaje (EVA)

\begin{tabular}{|l|l|l|}
\hline Estrategias & Sí & No \\
\hline Clases grabadas magistrales & $100 \%$ & \\
\hline Estudio de caso integrador & $100 \%$ & \\
\hline Foros y Chat & $100 \%$ & \\
\hline Cuestionarios cortos tipo Kahoot & $80 \%$ & $20 \%$ \\
\hline Ejercicios prácticos & $71,43 \%$ & $28,57 \%$ \\
\hline Portafolios digitales & $10 \%$ & $90 \%$ \\
\hline Glosario de términos & $100 \%$ & \\
\hline Tutorías académicas & $100 \%$ & \\
\hline Fuente: Alejo \& Fuentes (2020) & & \\
\hline
\end{tabular}

Fuente: elaboración propia

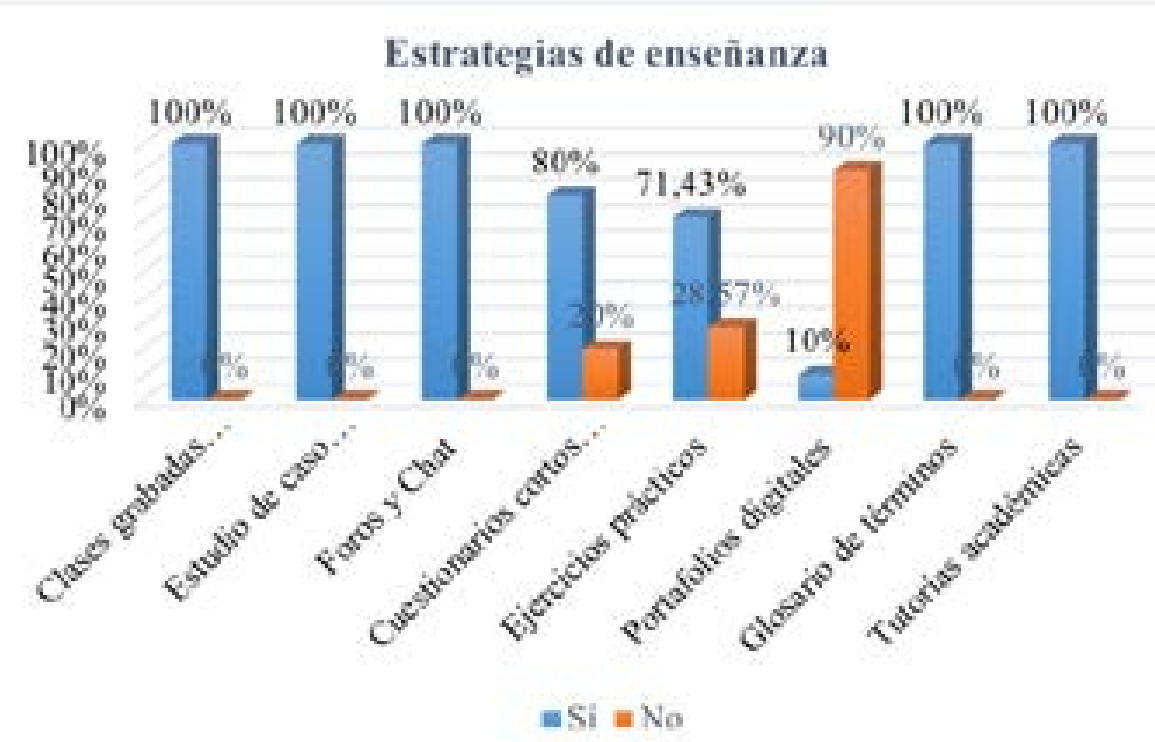

Figura 2. Estrategias de enseñanza

Fuente: Alejo \& Fuentes (2020)

Los resultados obtenidos (Figura 2) demuestran que de los profesores de la UISRAEL, el 100\% implementa diferentes estrategias para direccionar el proceso de enseñanza en el Entorno Virtual 
de Aprendizaje (EVA). Por ende, la educación actual exige a los profesores universitarios el dominio de las competencias digitales para un alto desempeño de su gestión académica.

Cabe señalar que, la competencia digital es el conjunto de conocimientos, habilidades, actitudes, estrategias y valores que se requieren cuando se emplean las TIC y los medios digitales para realizar tareas, resolver problemas, gestionar información, colaborar, crear, compartir contenidos, construir conocimiento de manera eficaz, eficiente, apropiada, crítica, creativa, autónoma, flexible, ética, y reflexiva (Ferrari 2012, citado por Esteve, 2016).

\section{Conclusiones}

La educación superior debe estar acorde con el desarrollo de los avances de la ciencia y tecnología para aprovechar las diversas bondades que brindan las TIC en el ámbito educativo, en concordancia a los requerimientos de los estudiantes de esta generación que son nativos de la era digital. Por ello, se requiere la implementación de estrategias de enseñanza con herramientas tecnológicas que facilitan diversos formatos en multimedia e hipertextos para la gestión del aprendizaje autónomo y el trabajo colaborativo en aprender uno de otros.

A la vez, desarrollar habilidades investigativas, promover el pensamiento autocritico y crítico en un proceso reflexivo, fomentar el pensamiento de resolución de problemas para la toma de decisiones acertadas, la administración efectiva del tiempo, y la participación en una experiencia inolvidable, amena, productiva, y en un dialogo entre iguales. Aprovechando oportunamente la comunicación sincrónica y asincrónica en aras de organizar los contenidos y las actividades que se llevaran a cabo en el quehacer educativo.

En síntesis, la planificación de estrategias de enseñanza genera un conjunto de procedimientos para planear a tiempo la toma de acciones en esos procedimientos que debe realizar el profesorado en la aplicación de métodos, técnicas, medios o recursos con el propósito de efectuar el proceso instruccional con rigor académico y de excelencia educativa, con el objetivo de desarrollar todas las actividades de un contenido específico. A modo de cierre, las estrategias de enseñanza se definen como los procedimientos que el agente de enseñanza utiliza en forma reflexiva y flexible para promover el logro de aprendizajes significativos en los alumnos (Díaz et al., 1999). 


\section{Referencias}

Arias, F. G. (2006). El proyecto de investigación. Introducción a la metodología científica. Caracas, Venezuela. Sexta Edición. Editorial: Episteme.

Ausubel, D. P., Novak, J., Hanesian, H. (1983). Psicología educativa: un punto de vista cognitivo. México. DF: Trillas.

Bennett, N. (1979). Estilos de enseñanza y progreso de los alumnos. Madrid: Morata.

Buontempo, M. P. (2017). La Enseñanza en la Era Digital. Una guía para la enseñanza y el aprendizaje. Virtualidad, Educación y Ciencia, 8(15), 190-192. https://revistas.psi.unc.edu.ar/index.php/vesc/article/ view/18968

Camacho, P. (2009). Metodología PACIE en los ambientes virtuales de aprendizaje para el logro de un aprendizaje colaborativo. file:///C:/Users/PC/Downloads/Dialnet-MetodologiaPACIEEnLosAmbientesVirtualesDeAprendiza-4156135.pdf

Collins, A. (1997). El potencial de las tecnologías de la información para la educación. Editorial. Pirámide. España.

Contreras, M. E., Leal, J., \& Salazar, R. (1999). Educación abierta ya distancia. Alternativa de autoinformación para el nuevo milenio. Bogotá: UNAD, 79.

Díaz, B. F. \& Hernández, R. G. (1999). Estrategias docentes para un aprendizaje significativo. Editorial Mc Graw. México. http://prepatlajomulco.sems.udg.mx/sites/default/files/estrategias de aprendizaje.pdf

Escamilla, J. (2019). El valor de enseñar para el mañana. Revista Telos. La educación en la era digital. Telefonía fundación. Madrid. https://dialnet.unirioja.es/servlet/articulo?codigo=7424073

Escontrela, M. R. \& Stojanovic, L. C (2004). La integración de las TIC en la educación: Apuntes para un modelo pedagógico pertinente. Revista de pedagogía. Caracas. Venezuela. http://ve.scielo.org/scielo.php?scrip$\underline{\mathrm{t}=\text { sci } \text { arttext\&pid=S0798-97922004000300006 }}$

Escudero, J. M. (2006). La formación del profesorado y la garantía del derecho a una buena educación para todos. Barcelona: Octaedro.

Escudero, J. M. (2008). Las competencias profesionales y la formación universitaria: posibilidades y riesgos. Revista de Docencia Universitaria. https://doi.org/10.4995/redu.2008.6267 
Esteve, M. F., Gisbert, M., Lázaro, J. (2016). La competencia digital de los futuros docentes: ¿cómo se ven los actuales estudiantes de educación? Perspectiva Educacional, Formación de Profesores. Pontificia Universidad Católica de Valparaíso. Chile. https://www.redalyc.org/pdf/3333/333346580004.pdf

Fernández, M. E., Ordoñez E., Morales, C.B., López, B.J. (2019). La competencia digital en la docencia universitaria. Colección universidad. Octaedro, S.R. Barcelona.

Fernández, P. A. (2007). Las plataformas e-learning para la enseñanza y el aprendizaje universitario en Internet. Universidad Complutense de Madrid. https://eprints.ucm.es/10682/1/capituloE learning.pdf

Galvis, A.H. (1992). Ingeniería de Software Educativo. Ediciones Uniandes.

González, M. D. (2002). Las Dificultades de aprendizaje en el aula. Departamento de psicología. Universidad de Cádiz. https://sid.usal.es/idocs/F8/FDO9786/gonzalez manjon.pdf

González, M. I. (2014). Los entornos virtuales como espacio de enseñanza-aprendizaje. Universidad Autónoma de México. Facultad de Estudios Superiores. Acatlán. México. http://132.248.9.195/ptd2014/ mayo/0713290/0713290.pdf

Guiza E. M. (2011). Trabajo colaborativo en la web: entorno virtual de autogestión para docentes. Tesis Doctoral. Universidad Autónoma de Baja California (UABC). https://www.tdx.cat/bitstream/handle/10803/59037/ tmge1de1.pdf? sequence=1\&isAllowed=y

Hernández, S. R., Fernández, C., \& Pilar, B. L. (2014). Metodología de la Investigación. SextaＥ～d i ción. MéxicoDF. McGrawHill. https://periodicooficial.jalisco.gob.mx/sites/periodicooficial.jalisco. gob.mx/files/metodologia de la investigacion - roberto hernandez sampieri.pdf

Instructivo de estructura tecno-pedagógica de aula virtual en el entorno virtual de Aprendizaje "EVA" UISRAEL 2020. (2020). https://uisrael.edu.ec/wp-content/uploads/2020/09/Instructivo-de-Estructura-de-Aula-Virtual-en-EVA-UISRAEL-24082020-v.1.2.pdf?x23864

MCA School of Business \& Postgraduate. (2020). Estrategias de enseñanza clase: Enseñanza activa para el autoaprendizaje y el aprendizaje colaborativo. Material propiedad de MCA School Higher Education 2.

Mestre, G. U., Valdés, T. P., Fonseca, P. J. (2007). Entornos virtuales de enseñanza aprendizaje. Ministerio de Educación Superior. Editorial universitaria. EDUNIV. El vedado. Ciudad de la Habana. https://libros.metabiblioteca.org/bitstream/001/251/8/978-959-16-0637-2.pdf

Mortera, G. (2007). El aprendizaje híbrido o combinado (Blended Learning): Acompañamiento tecnológico en las aulas del siglo XXI. Ciudad de México, México: Limusa. 
Ortiz, D. (2015). El constructivismo como teoría y método de enseñanza. Sophia, Colección de filosofía de la educación. Universidad Politécnica Salesiana. Cuenca. Ecuador. Recuperado en: https://www.redalyc. org/pdf/4418/441846096005.pdf

Pelayo, C. (2019). Tecnologías en las aulas. El desafío de la universidad para preparar los talentos del futuro. Revista Telos. La educación en la era digital. Telefonía fundación. Madrid. Recuperado en: https://telos. fundaciontelefonica.com/wp-content/uploads/2019/04/telos-110-enlighted-analisis-pelayo-covarrubias.pdf

Plataforma de las Ciencias del Aprendizaje. (s/f). Transformación educativa en línea. Herramientas para la enseñanza en línea. Transformación educativa en línea. https://thelearningsciences.com/herramientas-para-la-ensenanza-en-lineal

Real, J. (2011). Modelos educativos en el uso de las TIC. [Presentación en línea]. Recuperado en: https://es.slideshare.net/realjulio/modelos-educativos-en-el-uso-de-las-tic

Romero, F. A. (2017). Procedimiento para la evaluación del impacto de la formación de los docentes universitarios. Congreso Internacional "Ciencia, sociedad e investigación universitaria. Universidad Regional Autónoma de los Andes. https://repositorio.pucesa.edu.ec/handle/123456789/2058

Scheuermann, F., \& Barajas, M. (2003). Aspectos pedagógicos de la enseñanza y el aprendizaje en la red. La tecnología educativa en la enseñanza superior. Entornos virtuales de aprendizaje. Madrid: McGraw Hill.

Serrano, J. M., \& Pons, R. M. (2011). El constructivismo hoy: enfoques constructivistas en educación. Redie, 13(1). http://www.scielo.org.mx/scielo.php?pid=S1607-40412011000100001\&script=sci arttext

Universidad Tecnologica Israel. (2017). Modelo Educativo 2016-2020. https://uisrael.edu.ec/wp-content/ uploads/2019/10/Modelo Educativo UISRAEL_2016 2020-1-min.pdf 
Copyright (c) 2021 Betty Pastora Alejo y Arian Fuente Aparicio

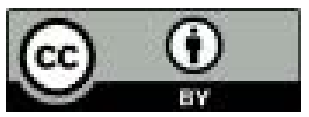

Este texto está protegido bajo una licencia internacional Creative Commons 4.0.

Usted es libre para Compartir-copiar y redistribuir el material en cualquier medio o formato - y Adaptar el documento - remezclar, transformar y crear a partir del material-para cualquier propósito, incluso para fines comerciales, siempre que cumpla las condiciones de Atribución. Usted debe dar crédito a la obra original de manera adecuada, proporcionar un enlace a la licencia, e indicar si se han realizado cambios. Puede hacerlo en cualquier forma razonable, pero no de forma tal que sugiera que tiene el apoyo del licenciante o lo recibe por el uso que hace de la obra.

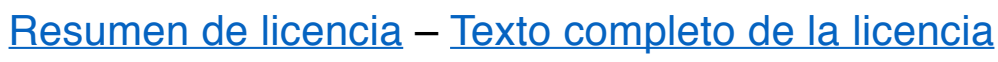

